\title{
The Effectiveness of Physical Therapy Following Neck Dissection: A Meta-Analysis
}

\author{
Yusuf M Agamawi*1,2, Masanari G Kato ${ }^{3,4}$, Shaun A Nguyen ${ }^{3}$ and Terry A Day ${ }^{3}$ \\ ${ }^{1}$ University of Minnesota Medical School, Minneapolis, US \\ ${ }^{2}$ Department of Otolaryngology -Head and Neck Surgery, Saint Louis University School of Medicine, US \\ ${ }^{3}$ Department of Otolaryngology - Head and Neck Surgery, Medical University of South Carolina, US \\ ${ }^{4}$ Department of Otolaryngology - Head and Neck Surgery, Ohio State University, US
}

*Corresponding author: Yusuf M Agamawi, Department of Otolaryngology-Head and Neck Surgery, Saint Louis University School of Medicine, 1008 South Spring Ave, 3rd Flr, St. Louis, MO 63110, US.

To Cite This Article: Yusuf M Agamawi, Masanari G Kato, Shaun A Nguyen, Terry A Day, The Effectiveness of Physical Therapy Following Neck Dissection: A Meta-Analysis. Am J Biomed Sci \& Res. 2021 - 12(2). AJBSR.MS.ID.001726. DOI: 10.34297/AJBSR.2021.12.001726.

Received: 眥 February 12, 2021; Published: 阱 March 03, 2021

\begin{abstract}
Purpose: Head and neck cancers (HNC) have high predilection of cervical lymph node metastases resulting in patients undergoing neck dissection (ND). Due to post-operative shoulder morbidity risks from ND, physical therapy (PT) has been used to address those complications, though studies are limited in demonstrating its efficacy. This study aims to evaluate the effects of PT on measures of shoulder pain and disability, quality of life, and shoulder mobility of patients that underwent ND as part of treatment for HNC.

Methods: Literature search was performed by two authors independently. Studies assessing effects of PT for HNC patients treated with ND were evaluated. Primary outcomes and measurements were Shoulder Pain and Disability Index (SPADI), Neck Dissection Impairment Index (NDII), and active shoulder abduction (ASA) in degrees.

Results: 108 patients from 3 studies. Effectiveness of PT on shoulder pain and disability, quality of life, and shoulder mobility were evaluated by SPADI, NDII, and ASA respectively. Pooled analyses of intervention (structured PT) versus control (standard, self-directed PT) mean differences (MD) for SPADI (MD=-8.13, 95\% CI [-14.06, -2.12], $\mathrm{p}=0.007$ ) and ASA (MD=24.74, 95\% CI [6.71,42.76], $\mathrm{p}=0.007$ ) favoured intervention, while NDII (MD=6.74, 95\% CI [-2.55,16.03], $\mathrm{p}=0.16$ ) favoured neither.

Conclusions: Rigorous therapy appears to provide greater benefits for shoulder pain, disability, and mobility. Further studies are warranted to characterize programs offering maximal support for this cohort of patients.

Implications for Cancer Survivors: Suggests that cancer survivors should be more aggressive towards physical therapy to improve shoulder function and pain after undergoing ND.
\end{abstract}

Keywords: Head and neck cancer, Neck dissection, Shoulder dysfunction, Disability, Pain, Physical therapy modalities

\section{Introduction}

Head and neck cancer (HNC) represents an increasingly common disease that is responsible for about 3\% of all malignancies, corresponding to a total of nearly 62,000 patients affected annually and 13,000 annual deaths in the United States [1]. These cancers often originate in the upper aerodigestive tract and metastasize to the neck requiring treatment with radiation, chemoradiation, and/ or surgery. Neck dissection (ND) is a therapeutic and diagnostic pro cedure currently utilized to remove diseased cervical lymph nodes. Depending on the type and extent of ND, patients may experience significant post-operative morbidity most commonly in the form of neck and shoulder stiffness, numbness, fibrosis, discomfort with and reduction in range of motion, and weakness.

The association of shoulder morbidity, namely pain and decreased range of motion, following ND is well established and must 
be addressed given its identified impact on patients' quality of life $[2,3]$. Although there has been a recent shift from radical ND to modified radical and selective ND to spare the spinal accessory nerve, shoulder complaints are still prevalent in $18-77 \%$ of modified radical and $29-39 \%$ of selective ND patients, and shoulder dysfunction in around $22 \%$ of spinal accessory nerve sparing ND patients [4-6]. Due to the spinal accessory nerve being responsible for innervation of the trapezius muscle, damage to the structure may result in shoulder dysfunction manifesting as decrease or loss of abduction, pain, girdle depression, trapezius atrophy, and scapular dyskinesia. If left untreated, it could ultimately lead to adhesive capsulitis and shoulder contractures [6,7].

The role of physical therapy (PT) has become pertinent in the post-operatively treatment of HNC patients following ND, with or without spinal accessory nerve preservation, due to its potential in decreasing shoulder pain, maintaining and improving range of motion, and preventing secondary disorders, such as adhesive capsulitis $[6,8]$. Although the benefits of PT are recognized, its current utilization is not standard. For example, the modalities of postoperative PT employed to prevent shoulder dysfunction can range from self-directed exercises to structured sessions or programs. Furthermore, among these programs, specifications regarding type of exercise, load, frequency, number of repetitions, duration, and goals vary widely. New efforts to synthesize literature since the Cochrane review by Carvalho et al. in 2012 is needed to further expand upon the effects and benefits of PT, and therefore, the focus of this review is to evaluate functional and quality of life outcomes of HNC patients who underwent ND and subsequent post-operative PT as a part of their treatment regimen [9].

\section{Methods}

\section{Data Sources and Searches}

Literature search was performed by 2 independent authors (Y.M.A. and M.G.K) using the PubMed and Scopus databases with the following phrases, respectively: ("Adult" [Mesh]) AND ("Head and Neck Neoplasms/surgery" [Mesh] OR "Neck Dissection" [Mesh]) AND ("Exercise Therapy" [Mesh] OR "Physical Therapy Modalities" [Mesh] OR "Rehabilitation OR Physical and Rehabilitation Medicine" [Mesh]); (neck dissection OR shoulder dysfunction) AND (physical therapy OR rehabilitation OR exercise OR physiotherapy). The results were screened by publication year, 2000 to July 26th, 2016, and limited to articles written in the English language. The remaining articles were screened for relevance by title and abstract independently, then cross-referenced between authors to determine eligibility for review. PRISMA literature search strategy is presented in Figure 1.

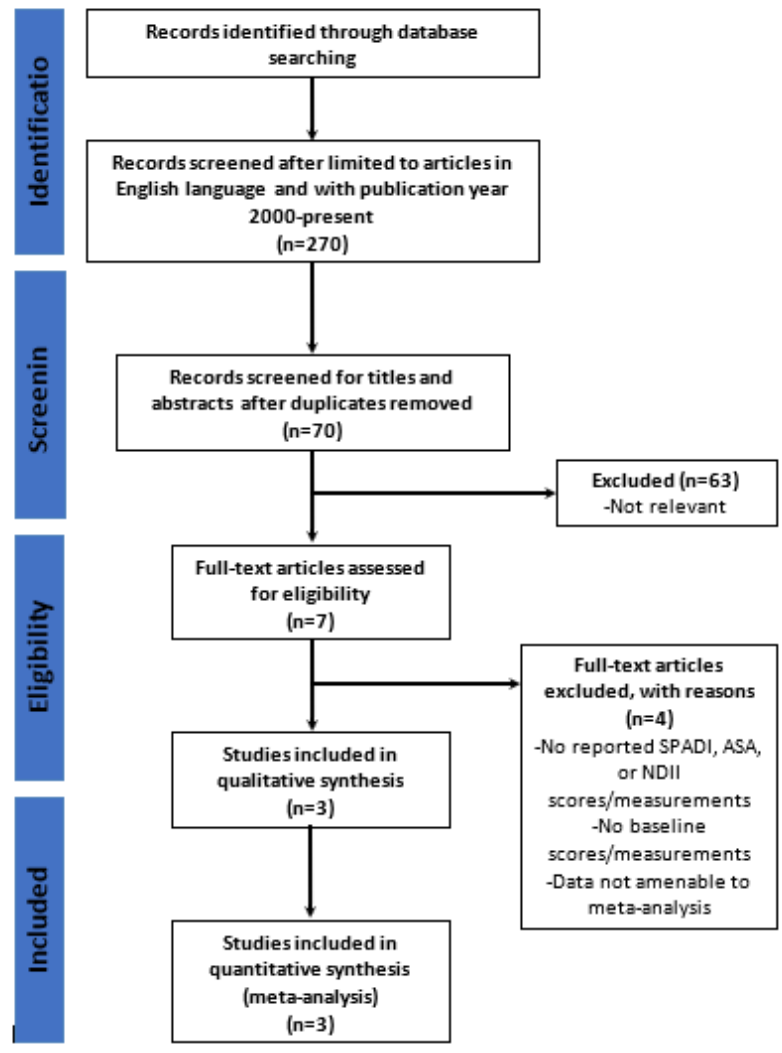

Figure 1: PRISMA study flow diagram outlining search strategy. SPADI, Shoulder Pain and Disability Index; NDII, Neck Dissection Impairment Index; ASA, active shoulder abduction. 


\section{Study Selection}

Inclusion criteria were as follows: studies pertaining to adults ( $\geq 18$ years of age), who underwent ND, as either a single or as part of multimodal treatment for HNC, and post-operative PT as treatment for ND side effects and/or complications, with pre- and postPT measurements of Shoulder Pain and Disability Index (SPADI) Neck Dissection Impairment Index (NDII), and/or active shoulder abduction (ASA) in degrees. These outcome measures were selected for relevance, validity, and consistency in the current literature. SPADI and NDII are validated tools in assessing shoulder pain, disability, and quality of life. The former index is utilized internationally for a variety of clinical conditions and contexts outside of HNC patients to quantify shoulder pain and functionality [10,11]. NDII on the other hand is an index specifically developed and aimed to assess the effect of ND on the quality of life of patients [12]. These validated indices combined provide a more comprehensive, general, and specific, analysis to quantify the functional status of the current patient cohort. Furthermore, shoulder syndrome, which is characterized by shoulder pain, winged scapula, and impaired shoulder abduction is one of the most significant functional complications of NDs. Although shoulder pain and having a winged scapula are points of great concern, limited shoulder abduction will inevitably affect patient capacities to fulfill activities of daily living (ADLs) such as washing hair and changing light bulbs [13]. Lastly, these measurements have been used frequently in the current literatures, particularly when studying the HNC patient cohorts, and therefore selected for the purposes of the current review.

\section{Data Extraction and Quality Assessment}

Data from included studies were independently extracted by 2 authors and then compared to confirm for accuracy. Additionally, the Sterne and Egger tests were performed for further assessment of risk of publication bias $[14,15]$. The outcomes of interest were pre- to post-PT changes in SPADI score, NDII score, and ASA degree for the control and intervention group. The mean and standard deviation for each outcome were obtained and a weighted average from each article was determined, which were utilized for further analysis.

\section{Data Synthesis and Analysis}

The meta-analysis used pre-PT (baseline) to post-PT measures to compare the intervention group (structured PT program) versus the control group (standard, self-directed PT exercises). Meta-analysis of selected studies with a continuous measure (comparison of means and standard deviations) was performed with Cochrane Review Manager (RevMan) version 5.3 (Nordic Cochrane Centre, Cochrane Collaboration, 2011, Copenhagen, Denmark). Both the fixed effects model and the random effects model were used in this study. Under the fixed effects model, it is assumed that all studies come from a common population, and that the effect size (standardized mean difference [SMD]) is not significantly different among the different trials. This assumption is tested by the heterogeneity test or I2. If this test yields a low probability value $(\mathrm{P}<.05)$, then the fixed effects model may be invalid. In this case, the random effects model may be more appropriate, in which both the random variation within the studies and the variation between the different studies are incorporated. Under the random effects model, the true effects in the studies are assumed to vary between studies, and the summary effect is the weighted average of the effects reported in the different studies [16]. The random effects model provides a more conservative estimate (i.e., with a wider confidence interval [CI]), but the results from the 2 models usually agree when there is no heterogeneity. When heterogeneity was present, the random effects model was the preferred model. For this study, the null hypothesis was that there was no difference between baseline and post-PT. Data are presented as mean \pm standard deviation $(95 \% \mathrm{CI})$ in this text and as mean difference (MD) in the figures. The total MD with $95 \% \mathrm{CI}$ is given for both the fixed effects model and the random effects model. If the value 0 is not within the $95 \% \mathrm{CI}$, then the SMD is statistically significant at the $5 \%$ level $(\mathrm{P}<.05)$.

Table 1: Articles satisfying inclusion criteria.

\begin{tabular}{|c|c|c|c|c|c|c|c|}
\hline $\begin{array}{l}\text { First Author } \\
\quad \text { (Year) }\end{array}$ & Study Type & $\begin{array}{l}\text { Level of } \\
\text { Evidence } \\
\text { (GRADE) }\end{array}$ & Groups & $\mathrm{n}$ & $\begin{array}{l}\text { Physical Therapy Reg- } \\
\text { imen }\end{array}$ & Selection Criteria & Adverse Events \\
\hline \multirow[t]{2}{*}{$\begin{array}{c}\text { McGarvey } \\
\text { (2015) }\end{array}$} & \multirow[t]{2}{*}{$\begin{array}{l}\text { Single blind } \\
\text { prospective } \\
\text { randomized } \\
\text { controlled } \\
\text { trial }\end{array}$} & High & Control & 17 & $\begin{array}{l}\text { Current usual physical } \\
\text { therapy care in Australia } \\
\text { after neck dissection } \\
\text { surgery for } 12 \text { weeks }^{\mathrm{a}}\end{array}$ & \multirow{2}{*}{$\begin{array}{l}\text { Inclusion: age } \geq 18 \text { years, } \\
\text { diagnosed with carcino- } \\
\text { ma of the head and neck } \\
\text { region, undergone ND with } \\
\text { accessory nerve preser- } \\
\text { vation and within past } 8 \\
\text { weeks before study entry, } \\
\text { with demonstrated clinical } \\
\text { signs of accessory nerve } \\
\text { shoulder dysfunction after } \\
\text { surgery, fully healed ND, } \\
\text { and ability to sufficiently } \\
\text { communicate in the English } \\
\text { language. }\end{array}$} & \multirow[t]{2}{*}{$\begin{array}{l}1 \text { intervention partic- } \\
\text { ipant died in the in- } \\
\text { tervention period, and } \\
\text { another participant } \\
\text { developed increasing } \\
\text { shoulder pain at } 3 \\
\text { weeks and the exercise } \\
\text { protocol was ceased. }\end{array}$} \\
\hline & & & $\begin{array}{l}\text { Inter- } \\
\text { vention }\end{array}$ & 22 & $\begin{array}{l}1 \text { supervised session } \\
\text { and } 2 \text { home sessions per } \\
\text { week for } 12 \text { weeks }\end{array}$ & & \\
\hline
\end{tabular}




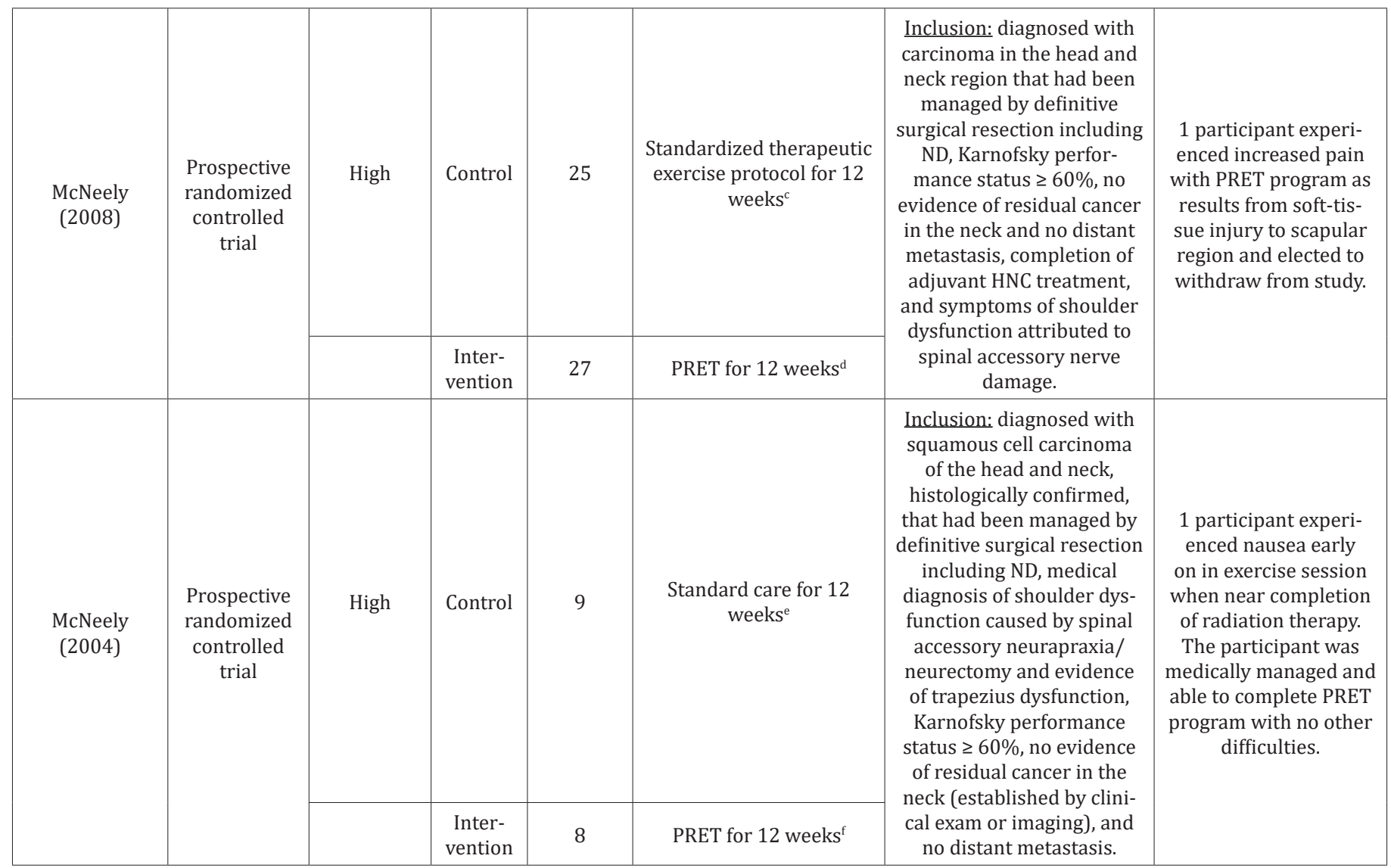

Note*: GRADE = Grading of Recommendations, Assessment Development and Evaluations; $\mathrm{n}=$ sample size; $\mathrm{SD}=$ standard deviation; $\mathrm{ND}=$ neck dissection; $\mathrm{HNC}=$ head and neck cancer; $\mathrm{PRET}$ = progressive resistance exercise training

${ }^{a}$ Combination of general advice and a brochure of generalized shoulder and neck exercises; brochure consisted of instructions and photographs of active-assisted glenohumeral joint exercises, active cervical spine range of movement exercises, advice about scar tissue massage and correct posture, and encouraging functional use of the upper limb.

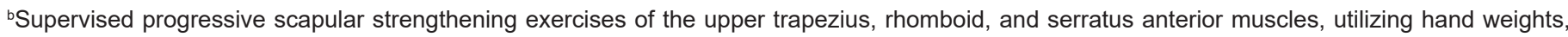
with the lowest possible weight being 0.5 kilograms.

'Supervised active and passive range of motion $(\mathrm{ROM}) /$ stretching exercises, postural exercises, and basic strengthening exercises with light weights (1-5 kilograms) and elastic resistance bands. Focus on rhomboids/middle trapezius, levator scapula/upper trapezius, biceps, triceps, deltoid, and pectoralis major.

dSame active and passive ROM/stretching exercises and postural exercises as standardized therapeutic exercise protocol (c), but progressive resistance exercise training (PRET) program replaced basic strengthening exercises and was tailored to each patient based on baseline testing results and was prescribed with intent to provide progressive overload to the specific muscle groups.

${ }^{e}$ Consisted of active and passive ROM exercises and stretching exercises.

${ }^{f}$ Exercised 3 times per week, progressive resistance exercise training (PRET) program was individualized to suit each participant; a physical therapist and/or physical therapy assistant supervised all exercise-training sessions (33-35 total sessions); series of 6 exercises with goal of enhancing scapular stability and restoring/maintaining the strength of the upper extremity; program was progressive in terms of number of sets, repetitions, and amount of weight lifted.

\section{Results}

Following inclusion and exclusion criteria, 3 articles were included in the analysis [17-19]. Detailed results of the literature search including levels of evidence using the Grading of Recommendations, Assessment, Development and Evaluations (GRADE) system are displayed in Table 1 [20]. While several studies were contextually relevant to the present study, they were unfortunately not included, many times due to the lack of measurement out- comes of interest (SPADI, NDII, active shoulder abduction). Notably, a study by Lauchlan, et al. included in the review by Carvalho et al., was not included for this reason [9,21]. All 3 included studies were prospective, randomized controlled studies. The study by McGarvey et al. was noted to be single-blinded [17]. These studies were found to present a low risk of bias (Figure 2), and moreover, the I2 statistic of $0 \%$ suggested non-significant study heterogeneity. Thus, a meta-analysis of these studies was performed utilizing the fixed-effect models. 


\section{Patient Demographics and Follow-up}

A total of 108 HNC patients, who underwent ND and post-operative PT were included. The pooled average age of participants of the 3 studies was 55.7 years old (range: 18-80 years-old), with $73.5 \%$ of participants being males. There was no statistical difference in age or sex representation among the 3 studies. Follow-up for the 2 McNeely et al. studies was at 3 months and for the McGarvey et al. study at 3, 6, and 12 months [17-19]. Therefore, data analysis between the studies was only considered at the 3-month follow-up for consistency.

\section{Shoulder Pain and Disability Index (SPADI)}

All 3 studies, totaling 108 patients, measured pre- and post-PT SPADI scores. Mean difference (MD) between the intervention and control groups revealed a statistically significant lower mean over- all SPADI score in the intervention group by -8.13 (95\% CI [-14.06, -2.12], $\mathrm{p}=0.007$ ) (Figure 3A) [17-19].

\section{Neck Dissection Impairment Index (NDII)}

2 studies, totaling 91 patients, measured pre- and post-PT NDII scores. Comparison of means between intervention and control groups revealed no statistical difference in NDII scores (MD $=6.74$, 95\% CI [-2.55,16.03], p=0.16) (Figure 3B) $[17,19]$.

\section{Active Shoulder Abduction (ASA)}

2 studies, totaling 56 patients, measured pre- and post-PT ASA degree measurements. Difference of means between the intervention and control groups revealed a statistically significant, higher mean of ASA degree in the intervention group by 24.74 degrees (95\% CI [6.71,42.76], $\mathrm{p}=0.007$ ) (Figure 3C) $[17,18]$.

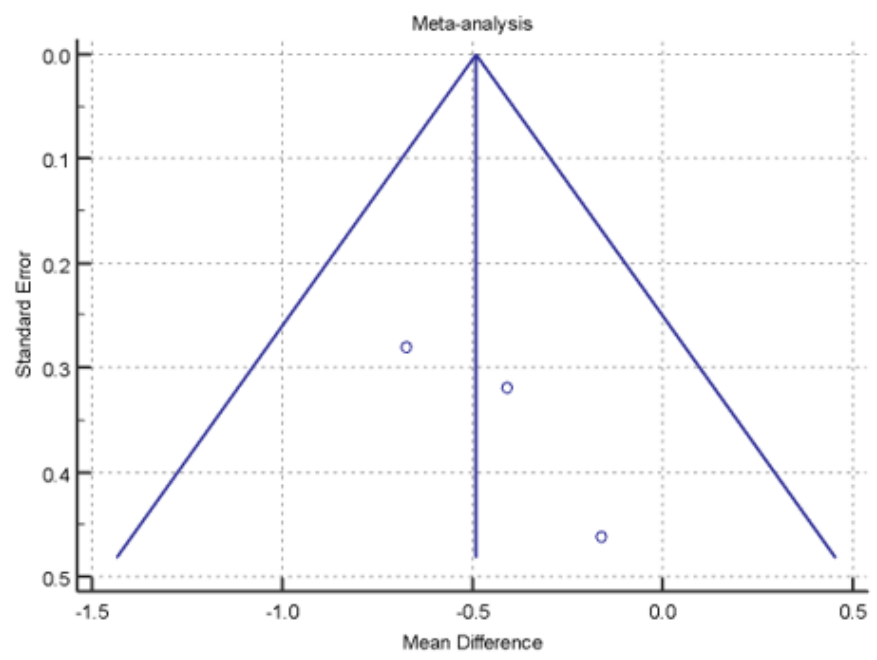

Figure 2: Funnel plot evaluating risk of publication bias in included studies.

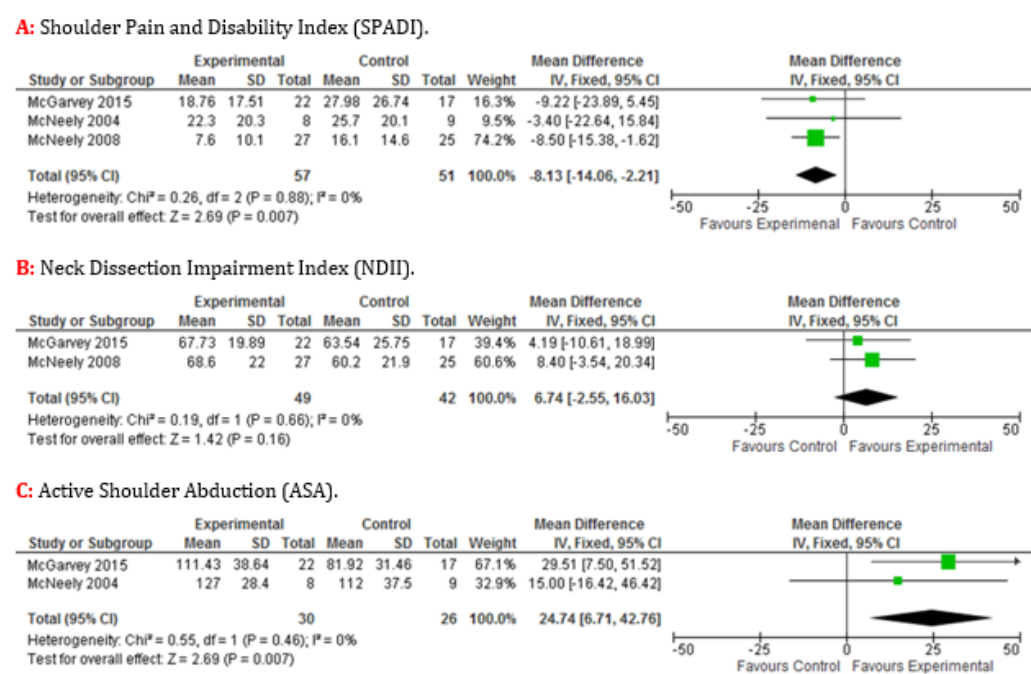

Figure 3: Forest plots of Shoulder Pain and Disability Index (SPADI) (3A), Neck Dissection Impairment. 


\section{Discussion}

The progressive utilization and involvement of post-operative PT in HNC patients treated with ND is often discussed among HNC surgeons for improving the likelihood of patient recovery, regaining functionality, and preserving quality of life. However, there is limited evidence and studies in the medical literature on its effectiveness. The present study is currently the largest and most recent meta-analysis addressing this topic. Although a similar analysis was conducted by Carvalho et al. in 2012, the study was limited to two studies, while this analysis contributes an additional study with a large cohort of 39 patients $[9,17]$. Not only does this add greater power to this analysis, but it also expands to include a different PT regimen to allow for more generalized application of results.

The first measured outcome is the SPADI score. The 100-point SPADI scoring system is a valid and reliable questionnaire that is responsive to change in clinical status and is used to assess for shoulder pain and disability [22,23]. The higher the SPADI score, the greater the degree of shoulder impairment. This meta-analysis identified that both the intervention and the control groups had a reduction in SPADI scores at the 3-month follow-up, with the magnitude of this decrease greater in the intervention group (MD = 8.13; 95\% CI [-14.06, -2.12], $\mathrm{p}=0.007$ ). Paul et al. demonstrated that a change in as little as 8 points on the scoring system was associated with clinical significance perceived by patients [23]. This indicates that PT in general is beneficial, however, a more structured PT program may provide greater and perceivable benefit in alleviating shoulder pain and disability.

The second measured outcome, NDII, is an instrument utilized to assess quality of life. The NDII is a reliable 10-question tool, scored from 1 ("a lot") to 5 ("not at all"), that is scaled to a 100-point cumulative score that has been validated for assessing impairment following ND [12]. The higher the score, the lesser the impairment to quality of life. The analysis showed an increase in the mean NDII scores in both groups at 3 months, and although the results favored the intervention group, it was not statistically significant (MD of 6.74; 95\% CI [-2.55,16.03], $\mathrm{p}=0.16$ ). This demonstrates that while both self-directed PT and structured PT may improve quality of life, one is not superior to the other. This result may be due to the small sample sizes, and thus a greater-powered study may detect a significant difference between self-directed and structured PT approaches.

The third measured outcome is the degrees of ASA. The number of ASA degrees is used as a marker for shoulder mobility, with increasing values correlating with greater shoulder mobility. Like the findings of the SPADI scores both the intervention and control groups had improvement in ASA degrees at roughly 3 months when compared to baseline, however the degree of improvement was significantly greater in the intervention group who received struc- tured PT (MD = 24.7; 95\% CI [6.71,42.76], $\mathrm{p}=0.007)$. This again suggests that although patient-directed exercises could improve outcomes, a structured PT program provides additional benefits regarding shoulder mobility ASA.

There are several notable limitations of this study with the most prominent being sample size and follow-up period. Though the present case includes an additional study compared to Carvalho et al., the sample size remains relatively small [9]. This warrants more studies investigating the benefits of more rigorous/structured PT regimens to elaborate on the role of PT in the standard of HNC patients following ND. Because two of the three studies had a single follow-up period at 3 months, and McGarvey et al. had multiple follow-up periods at 3,6 , and 12 months, this study only evaluated baseline measurements (pre-PT) in comparison to 3-month measurements (post-PT) [17-19]. Consequently, more long-term (6- and 12-month) measurements that were provided by McGarvey et al., were not incorporated in the analysis [17]. This was done to preserve consistency in measurement timing among the three studies given the McNeely et al. studies did not have follow-up data past 3 months $[18,19]$. Furthermore, the intervention programs in all the studies involved progressive strengthening exercises, and two of the three programs involved individualizing the program to better accommodate each participant [17-19].

Despite these limitations, the short follow-up period may imply that improvements can be achieved earlier with more structured PT programs. Moreover, this study groups specific PT modalities or regimens to the category of structured PT programs and may provide a more generalizable portrayal of structured PT benefits. It is noted with certainty that more studies are necessary to further characterize the varying PT modalities as well as regimens according to their differential impact on functional outcomes and quality of life in HNC patients treated with ND.

\section{Conclusion}

Shoulder pain, dysfunction, and impairment are pervasive in HNC patients who have undergone ND. Post-operative PT modalities have promising benefits with the present study showing that more rigorous and structured PT programs lead to greater improvement in shoulder pain, disability, and mobility when compared to selfdirected exercises. This was identified by observing greater improvements in SPADI scores and ASA degrees. Given the small number of studies included in this analysis, in addition to limited sample size, differing PT regimens, and heterogeneity in PT outcome measurements, it is recommended that additional studies are conducted to further characterize PT regimens to expand upon their potential impact on the recovery of this patient cohort.

\section{References}

1. Siegel RL, KD Miller, A Jemal (2016) Cancer statistics, 2016. CA Cancer J Clin 66(1): 7-30. 
2. Shah S, G Har El, RM Rosenfeld (2001) Short-term and long-term quality of life after neck dissection. Head Neck 23(11): 954-961.

3. Rogers SN, Alfio Ferlito, Phillip K Pellitteri, Ashok R Shaha, Alessandra Rinaldo (2004) Quality of life following neck dissections. Acta Otolaryngol 124(3): 231-236.

4. Leipzig B, J Y Suen, J L English, J Barnes, M Hooper (1983) Functional evaluation of the spinal accessory nerve after neck dissection. Am J Surg 146(4): 526-530

5. Dijkstra PU, P C van Wilgen, R P Buijs, W Brendeke, C I de Goede, et al. (2001) Incidence of shoulder pain after neck dissection: a clinical explorative study for risk factors. Head Neck 23(11): 947-953.

6. Bradley PJ, Alfio Ferlito, Carl E Silver, Robert P Takes, Julia A Woolgar, et al. (2011) Neck treatment and shoulder morbidity: still a challenge. Head Neck 33(7): 1060-1067.

7. Kelley MJ, TE Kane, BG Leggin (2008) Spinal accessory nerve palsy: associated signs and symptoms. J Orthop Sports Phys Ther 38(2): 78-86.

8. Lauchlan DT, JA McCaul, T McCarron (2008) Neck dissection and the clinical appearance of post-operative shoulder disability: the postoperative role of physiotherapy. Eur J Cancer Care (Engl) 17(6): 542-548.

9. Carvalho AP, FM Vital, BG Soares (2012) Exercise interventions for shoulder dysfunction in patients treated for head and neck cancer. Cochrane Database Syst Rev (4): CD008693.

10. Torres Lacomba M, Beatriz Sánchez-Sánchez, Virginia Prieto-Gómez Soraya Pacheco-da-Costa, María José Yuste-Sánchez, et al. (2015) Spanish cultural adaptation and validation of the shoulder pain and disability index, and the oxford shoulder score after breast cancer surgery. Health Qual Life Outcomes 13: 63.

11. Vrouva S, C Batistaki, E Koutsioumpa, D Kostopoulos, E Stamoulis, et al. (2016) The Greek version of Shoulder Pain and Disability Index (SPADI): translation, cultural adaptation, and validation in patients with rotator cuff tear. J Orthop Traumatol 17(4): 315-326.

12. Taylor RJ, Judith C Chepeha, Theodoros N Teknos, Carol R Bradford, Pramod K Sharma, et al. (2002) Development and validation of the neck dissection impairment index: a quality of life measure. Arch Otolaryngol Head Neck Surg 128(1): 44-49.

13. Khadilkar L, Joy C MacDermid, Kathryn E Sinden, Thomas R Jenkyn, Trevor B Birmingham, et al. (2014) An analysis of functional shoulder movements during task performance using Dartfish movement analysis software. Int J Shoulder Surg 8(1): 1-9.
14. Egger M, G Davey Smith, M Schneider, C Minder (1997) Bias in metaanalysis detected by a simple, graphical test. BMJ 315(7109): 629-634

15. Sterne JA, M Egger (2001) Funnel plots for detecting bias in metaanalysis: guidelines on choice of axis. J Clin Epidemiol 54(10): 10461055.

16. Borenstein M, Larry V Hedges, Julian P T Higgins, Hannah R Rothstein (2009) Introduction to meta-analysis. Chichester pp. 421.

17. McGarvey AC, Gary R Hoffman, Peter G Osmotherly, Pauline E Chiarelli (2015) Maximizing shoulder function after accessory nerve injury and neck dissection surgery: A multicenter randomized controlled trial. Head Neck 37(7): 1022-1031.

18. McNeely ML, Matthew Parliament, Kerry S Courneya, Hadi Seikaly, Naresh Jha, et al. (2004) A pilot study of a randomized controlled trial to evaluate the effects of progressive resistance exercise training on shoulder dysfunction caused by spinal accessory neurapraxia/ neurectomy in head and neck cancer survivors. Head Neck 26(6): 518530.

19. McNeely M.L, Matthew B Parliament, Hadi Seikaly, Naresh Jha, David J Magee, et al. (2008) Effect of exercise on upper extremity pain and dysfunction in head and neck cancer survivors: a randomized controlled trial. Cancer 113(1): 214-222.

20. Atkins D, Dana Best, Peter A Briss, Martin Eccles, Yngve Falck Ytter, et al. (2004) Grading quality of evidence and strength of recommendations. BMJ 328(7454): 1490.

21. Lauchlan DT, J A McCaul, T McCarron, S Patil, J McManners, et al. (2011) An exploratory trial of preventative rehabilitation on shoulder disability and quality of life in patients following neck dissection surgery. Eur J Cancer Care (Engl) 20(1): 113-122.

22. Heald SL, DL Riddle, RL Lamb (1997) The shoulder pain and disability index: the construct validity and responsiveness of a region-specific disability measure. Phys Ther 77(10): 1079-1089.

23. Paul A, M Lewis, M F Shadforth, P R Croft, D A W M Van Der Windt, et al. (2004) A comparison of four shoulder-specific questionnaires in primary care. Ann Rheum Dis 63(10): 1293-1299. 